
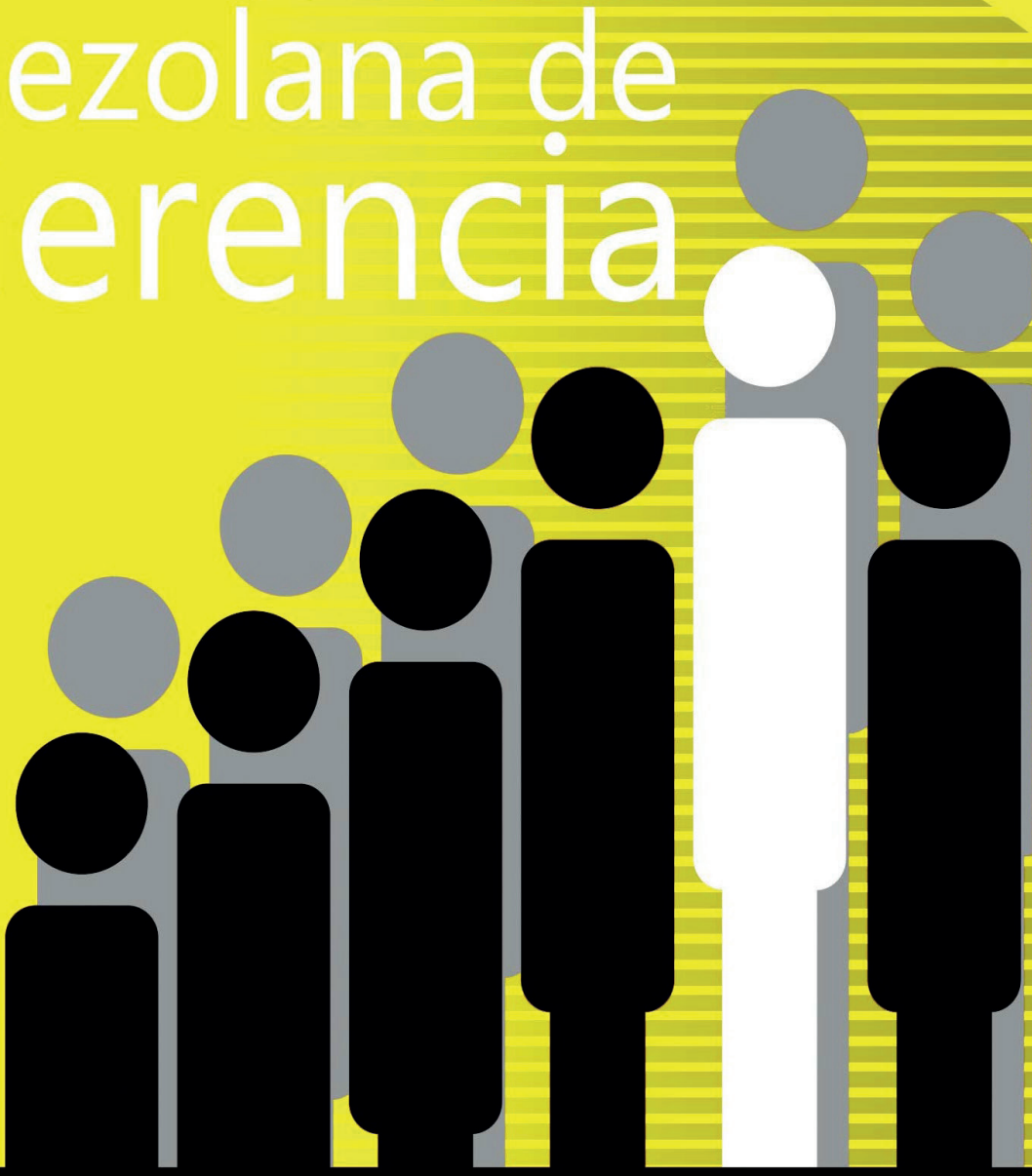


\title{
Innovación y Diseño: cómo transformar organizaciones y prosperar en mercados competitivos
}

\author{
Montenegro Orozco, Josué Carlos*
}

\section{Resumen}

El objetivo del presente artículo es analizar la estructura del modelo de innovación impulsado por procesos de diseño y como este puede promover transformaciones estratégicas en las firmas, que les permitan mantenerse competitivas en el mercado y responder de manera más efectiva a los desafíos que este impone. La investigación es de tipo descriptiva y ha seguido un proceso sistémico de revisión de literatura en bases de datos especializadas para identificar fuentes de información pertinentes. Se encontró que el Pensamiento de Diseño a través del impulso de prácticas metodológicas, restructuración de procesos, y fomento al intercambio de información y aprendizaje continuo puede transformar la cultura de una organización, permitiendo el surgimiento de nuevos métodos de trabajo que agilicen la identificación e interpretación de necesidades y problemas al igual que la formulación de acciones de respuesta. Se concluye que el modelo de innovación planteado puede tener un papel estratégico en las organizaciones en la medida en que también existan estructuras internas de apoyo.

Palabras Clave: pensamiento de diseño; innovación; cultura organizacional; ventajas competitivas

\section{Recibido: $15.10 .20 \quad$ Aceptado: 15.02.21}

* Master en Emprendimiento e Innovación Social por la London School of Economics and Political Sciences (LSE). Economista por la Pontificia Universidad Católica del Ecuador. Oficial de Estrategias de Innovación y Desafios de Diseño para el Laboratorio de Innovación Panda Labs del World Wildlife Fund (WWF). Email: josuemont0503@gmail.com. ORCID: https://orcid. org/0000-0001-9824-6372. 


\title{
Innovation and Design: How to Transform Organizations and Thrive in Competitive Markets
}

\begin{abstract}
This article analyzes how the design-driven innovation model works and how it can promote strategic transformations in firms allowing them to remain competitive in the market and respond more effectively to the challenges that it imposes. The research is descriptive and followed a systemic-review process of literature to identify relevant sources of information. The research found that Design Thinking can transform an organization's culture by promoting new methodological practices, restructuring processes, and fostering the exchange of information and continuous learning. In doing this, Design Thinking can promote the use of new work methods that speed up the identification and interpretation of users' needs and problems as well as encourage rapid strategic responses. It is concluded that the proposed innovation model can have a determinant role in organizations to the extent that internal support structures shore it up.
\end{abstract}

Key Words: Design Thinking, Innovation, Organizational Culture, Competitive Advantages

\section{Introducción}

En un mundo en constante ebullición e incertidumbre caracterizado por mayor competencia, globalización y evolución rápida en las necesidades e intereses de los consumidores, se exige de las empresas un cambio radical en sus operaciones, que les permita al mismo tiempo responder con rapidez, flexibilidad, eficiencia y un alto grado de innovación (Marín, 2004; Rizzo, Deserti, \& Cobanli, 2017). A pesar de ello y de los efectos que este cambio puede tener en cuanto a posicionamiento competitivo, participación en el mercado y sobrevivencia a largo plazo, ha sido limitado el accionar de las firmas por responder efectivamente a llamados de transformación y adaptación estratégica.
De hecho, conforme lo mencionan, Vega, et al, (2020) y Escorsa \& Pasola (2004), las firmas, en la mayoría de los casos, han reaccionado de manera reactiva a cambios en su sector, optando por modificaciones a su tamaño y estructura, que lejos de tener un impacto real han conllevado un uso ineficiente de capacidades y recursos (Junginger, 2008; Rosenberg, Chauvet, \& Kleinman, 2016).

En este sentido, se puede señalar que la capacidad de innovación de una firma y las ventajas competitivas que generen por ella van más allá de la implementación de nuevas tecnologías y restructuración de procesos. De acuerdo con Wyrwicka \& Chuda (2019:280), la innovación es resultado del conjunto de creencias, actitudes, comportamientos, y 
valores que mantienen las personas que forman parte de un organización.

En otras palabras, la innovación guarda estrecha relación con la cultura organizacional y esta última puede potencialmente mostrarse a favor o en detrimento de la primera. Especialmente graves son las circunstancias en que la cultura organizacional se muestra inflexible y no permite el desarrollo de nuevas habilidades gerenciales que hagan frente rápidamente a los cambios contextuales anteriormente mencionados (Schein \& Schein, 2019). En esta línea, resulta común que las organizaciones tropiecen al crear soluciones para sus usuarios al no poseer las destrezas necesarias para entender sus problemas y necesidades reales (Brown \& Wyatt, 2010a; Koen, 2015).

Frente a este contexto, se ha señalado la creciente importancia de implementar una cultura de diseño en las firmas como mecanismo de actualización y cambio cultural, que permita a través de sus procesos y programas mejorar la eficiencia de sus empleados en la identificación de problemas, generación de ideas, formulación de soluciones y aprendizajes rápidos con bajos costos de ejecución (Buchanan, 2015; Organisation for Economic Cooperation and Development, 2015; Perks, Cooper, \& Jones, 2005). Específicamente, la cultura de diseño, apalancada por procesos metodológicos como Lean Innovation y Design Thinking (Pensamiento de Diseño) se ha caracterizado por impulsar iniciativas de innovación disruptivas que abordan, por ejemplo, nuevos aspectos de la relación con los clientes, diferentes canales de distribución, redes de aliados y modelos de negocio a través del fomento al capital intelectual de la organización, entendido como la combinación de capital humano, relacional, y estructural (Kong, 2010) ${ }^{1}$.

A pesar de lo mencionado, es necesario reconocer que la implementación del pensamiento de diseño a nivel organizacional no es un proceso libre de obstáculos. En particular, sus limitaciones dentro de las firmas se han entrado en torno a falta de contextualización, mal entendimiento, e imposición vertical. De hecho, si bien el pensamiento de diseño busca impulsar una cultura de exploración y toma de riesgos, su efectividad depende en gran parte de la cultura actualmente presente en la organización. Si esta última es contraria a los postulados propios de este proceso, resulta evidente que cualquier intento de transformación estratégica será difícil de llevar (Boland Jr, Collopy, Lyytinen, \& Yoo, 2008; Chang, Kim, \& Joo, 2013). De igual manera, si el proceso es impuesto de manera vertical y no se estructura a partir de negociación y alineamiento con el resto de la organización, la cultura de diseño tomará fuerza solo en espacios aislados (Chiva \& Alegre, 2009; Filippetti, 2011). Finalmente, si la cultura de diseño es vista de manera reduccionista y considerada tan solo como fórmula, los efectos e impactos que genere su uso

1 El capital relacional se refiere a las relaciones que mantiene una organización con actores externos a la misma y a las oportunidades de intercambio de información, desarrollo de conocimientos, y generación de ideas de innovación que surgen como resultado El capital estructural, por su parte, se define como el conjunto de conocimientos que posee una organización y que se presentan en la forma de manuales de procedimiento, rutinas, publicaciones, patentes, etc. (Kong \& Thomson, 2009; Marr \& Roos, 2005). 
serán pequeños (Deserti \& Rizzo, 2014).

En línea con lo mencionado, y con el objetivo de promover un entendimiento acertado acerca de lo que implica impulsar una cultura de diseño y como esta puede fomentar procesos de innovación en una organización, esta investigación analizará en las siguientes secciones los modelos de innovación existentes, los fundamentos que dan forma a una cultura organizacional, que es el pensamiento de diseño, como puede su implementación dentro de una firma promover una cultura orientada a la innovación estratégica, y los obstáculos que esta implementación puede encontrar.

El presente estudio se sustenta en un proceso de revisión sistémica de literatura y toma la forma de una investigación descriptiva en tanto que busca caracterizar y evidenciar los efectos de un fenómeno de estudio específico (Hernández, et al, 1998). El proceso de revisión sistémica comprendió cuatro etapas. La primera de ella se estructuró en torno a la identificación de bases de datos y búsqueda preliminar de documentación, resultado de la cual se encontró que Scopus, Emerald, y Google Scholar respondían ampliamente las necesidades de la investigación, albergando más de 100 artículos científicos, libros y reportes técnicos de relevancia. Posteriormente, la documentación encontrada fue filtrada en base al alineamiento de su introducción, resumen y conclusiones con temas de atención clave, como pensamiento de diseño, innovación, ventajas competitivas, y cultura organizacional lo que permitió descartar 23 artículos cuyo abordaje no resultaba pertinente. Seguidamente, los documentos restantes fueron sometidos a un proceso de análisis de contenido que permitió codificar categorías de información según tópicos de relevancia, tales como elementos de la innovación, desafíos organizacionales, cultura organizacional y pensamiento de diseño, casos de estudio, limitaciones, entre otros.

\section{Modelos de Innovación}

La innovación puede entenderse como un fenómeno sistemático de cambio y transformación institucional que busca a través de la restructuración de procesos internos, incluidos aquellos de gobernabilidad, producción y comercialización, plantear nuevos productos y propuestas de valor, lograr eficacia en el uso de recursos, atender a segmentos de mercado diferentes, mejorar el servicio prestado a los clientes actuales, y últimamente constituir ventajas diferenciadoras que potencien la cuota de mercado de las firmas e impulsen su sobrevivencia a corto, mediano y largo plazo (Porter, 2007; Schumpeter \& Arrarte, 1976).

A un nivel más específico, la innovación también puede ser vista como un proceso de etapas integrado por mecánicas de identificación, desarrollo y lanzamiento comercial (Cooper, 2008). La relación entre las mismas, lejos de tener un entendimiento universal, ha sido ampliamente estudiada en los últimos años, resultado de los cual varios modelos que buscan su explicación han surgido, entre los cuales se destacan especialmente los siguientes, de acuerdo con Cooper (2008), Deserti \& Rizzo (2014), Koen (2015) y Vega et al,(2020):

- Modelo Lineal: Sostiene que la innovación se origina como resultado de un proceso secuencial fundamentado en la utilización 
planificada y control directo del conocimiento científico a través de 5 fases: investigación aplicada, desarrollo, producción, comercialización y mercado. Es decir, este modelo ve a la innovación como siendo el resultado de un proceso exacto de acoplamiento de partes, en el cual la empresa se apalanca del acervo técnico existente para impulsar procesos internos automáticos.

- Modelo de Marquis: Plantea que cualquier innovación surge de una idea técnicamente factible $y$ potencialmente comercializable. Si existe un buen entendimiento acerca de la idea se parte a la construcción de prototipos y a pruebas pilotos que permitan entender costos y procesos requeridos. Si el conocimiento es escaso, se realizan investigaciones de mercado. Finalmente, y luego de resultados de prueba satisfactorios con el potencial consumidor, se trabaja en el diseño, fabricación y mercadeo pertinentes.

- Modelo de Kline: Argumenta tal como el Modelo de Marquis que la innovación parte de una idea potencialmente atractiva para el mercado pero se diferencia al señalar que su desarrollo y posterior lanzamiento involucran un proceso permanente, no solo inicial, de retroalimentación y mejora continua.

- Modelo de Fases y Puertas: Consiste en 5 etapas iterativas y no secuenciales que buscan impulsar a una solución desde su descubrimiento, definición de alcance y relevancia, desarrollo, validación y lanzamiento. Al contrario de modelos anteriores plantea la evaluación constante del proceso después de cada etapa. Si el mismo es deficiente, no ha incorporado retroalimentaciones previas, y no muestra impacto, el proyecto es eliminado. Por el contrario, si el mismo cumple con indicadores de rendimiento, es impulsado $y$ más recursos son otorgados a su desarrollo. Puede correr el riesgo de trabarse ante procesos burocráticos, falta de riesgo, y ausencia de responsabilidad.

- Modelo de Innovación Impulsado por Procesos de Diseño: Conjuga varios elementos de los modelos anteriormente analizados tales como la búsqueda permanente de información, descubrimiento de problemas, construcción de prototipos, análisis de factibilidad y evaluación de impacto. Sin embargo, se diferencia significativamente de ellos, al orientarse al desarrollo de procesos de innovación de corte disruptivo y transformacional que buscan generar mejoras significativas a líneas de producción existentes 0 establecer nuevas redes de valor con clientes que aún no participan en el mercado objetivo.

Tales objetivos son conseguidos a través del trabajo a múltiples niveles de la organización. El macro nivel constituye la propia cultura organizacional, la misma que se busca transformar en una más abierta a través del desarrollo de un sistema de competencias y habilidades orientado al intercambio de conocimiento, impulso al capital intelectual, fortalecimiento de liderazgos internos, toma de riesgos, evaluación de impacto, alineamiento estratégico y aprendizaje constante. El micro nivel, por su parte, se nutre del nivel superior anteriormente mencionado y de las prácticas que con él se implementaron para identificar, interpretar y dar 
respuesta rápida a las necesidades y motivaciones de los usuarios internos o externos a los que se pretende servir. El resultado de todo esto es el repensamiento constante del modelo de negocio de la firma, y la formulación de procesos ágiles de ideación, desarrollo y comercialización que permitan explorar y aprovechar oportunidades de negocio no-tradicionales, diagrama 1.

\section{Diagrama 1 \\ Modelo de Innovación Impulsado por Procesos de Diseño}

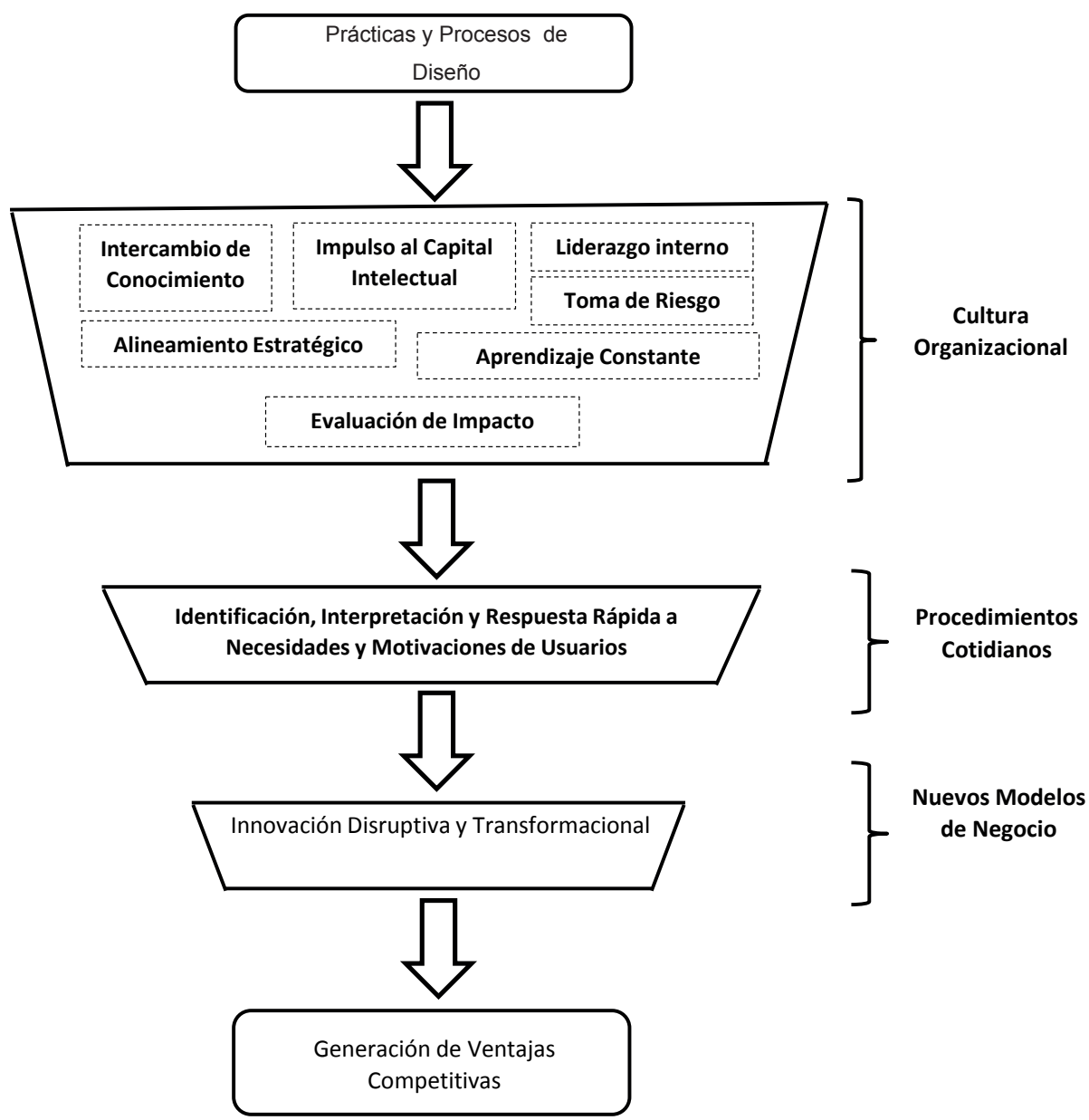

Fuente: Elaboració propia basada en Cooper (2008), Deserti \& Rizzo (2014), Koen (2015) y Vega et al, (2020) 


\section{Pensamiento de Diseño}

El pensamiento de diseño destaca por ser una de las prácticas y procesos más importantes en cuanto se refiere al fomento de una cultura de diseño y experimentación. La razón para aquello reside no tanto en sus metodologías y ejercicios sistémicos, como pueden ser las entrevistas etnográficas, la ideación conjunta de soluciones o la construcción de modelos de baja intensidad, pero en el razonamiento abductivo que recubre a cada una de estas actividades. Como se puede observar en el diagrama 2, este tipo de razonamiento resulta del uso conjunto y secuencial de procesos de divergencia (exploración) y convergencia (definición de hallazgos y lecciones) que de forma simultánea permiten analizar el contexto y dar respuesta a preguntas tales como: ¿Cuál es el problema que enfrentan los usuarios?, ¿Quién tiene este problema?, y ¿Cuál es el valor de solucionar el mismo?, considerando en sus análisis la observación cercana de su público objetivo (i.e., que hace, piensa, necesita y quiere). En otras palabras, y tal como lo describe Sanders (2002), el pensamiento de diseño busca transformar a la organización en una entidad que haga de su misión la búsqueda constante de nuevas fuentes de información, el desafío a explicaciones generalmente aceptadas y la inferencia de nuevas realidades y modelos de negocio.

A un nivel más específico y técnico, y como también lo muestra el Gráfico 2, se puede distinguir en el Pensamiento de Diseño, la existencia de ciclos iterativos de observación y análisis, concepción y prototipaje que buscan generar mayor entendimiento acerca de una situación a través de un filtro metodológico, sometiendo a problemas y potenciales ideas a evaluaciones permanentes hasta que estas últimas se puedan considerar soluciones y sostenerse en claros parámetros de viabilidad, factibilidad y deseabilidad comercial (Jayakumar, Das, \& Srivastava, 2019; Martin, 2009).

\section{Diagrama 2 \\ Pensamiento de Diseño: Proceso Sistémico}

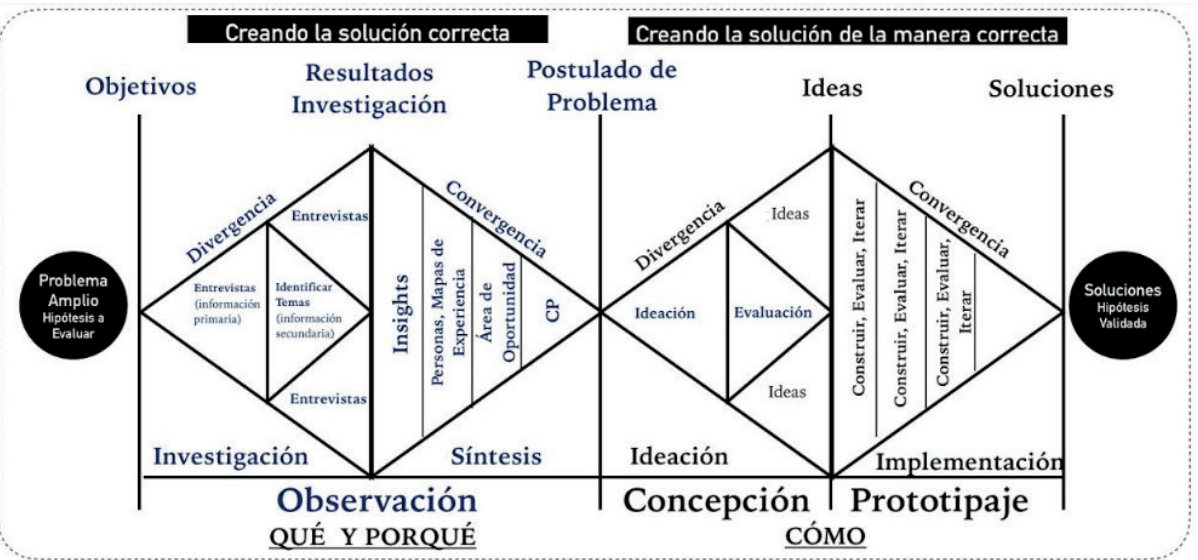

Fuente: Elaboración propia adaptado de Make Sense \& Schoolab (2020) 
Los ciclos anteriormente mencionados se enmarcan en cinco etapas: empatía, definición, ideación, prototipaje y testeo (Jayakumar et al, 2019). La etapa de empatía involucra la utilización de métodos tales como observaciones etnográficas y entrevistas y tiene como objetivo entender de cerca el pensamiento, sentimientos, contexto y rutinas de los usuarios (Dunne, 2011). La fase de definición, por otra parte, procura sistematizar la información anteriormente recogida, a través de métodos como el análisis temático, creación de personas y mapas de experiencia con el objetivo de definir detalladamente el problema a resolver (Dorst \& Cross, 2001).

El proceso de ideación, por su lado, propicia instancias multidisciplinarias de co-creación y diseño de ideas donde se utilizan variaciones de la técnica "lluvia de ideas" para crear posibles soluciones (Suciu \& Baughn, 2016). Finalmente, las etapas de prototipaje y testeo involucran un proceso conjunto en donde a través de réplicas de baja, media y alta fidelidad de las soluciones anteriormente ideadas se procura interactuar con usuarios/beneficiarios finales para identificar rápidamente y con un bajo costo, posibles errores, elementos destacables, y componentes complementarios que permitan atender correctamente el problema (Brown \& Wyatt, 2010b; Glen, Suciu, Baughn, \& Anson, 2015; Lawson, 2006; Liedtka \& Ogilvie, 2011).

En líneas generales, y como se mencionó al inicio de esta subsección, se puede decir que el Pensamiento de Diseño, si bien comúnmente entendido como la aplicación práctica de las etapas recién mencionadas, va más allá. Lo que realmente busca es familiarizar a la organización con un conjunto de técnicas que a través de su uso cotidiano generen nuevas dinámicas organizacionales.

\section{Pensamiento de diseño y cultura organizacional}

Aunque sea un concepto elusivo, entender la cultura organizacional de una firma resulta fundamental para la implementación de un programa de Pensamiento de Diseño que busque fomentar mayor innovación. En este sentido, la misma podría definirse como la conjunción de tres niveles o capas que se refuerzan entre sí: artefactos observables, normas y valores, y supuestos básicos fundamentales (Schein, 2017). Los artefactos observables, como su nombre lo indica, se refieren a elementos fácilmente identificables, tales como símbolos, estructuras físicas, lenguaje, rutinas y ceremonias. Las normas y valores, por otro lado, involucran las pautas abiertamente favorecidas por la organización a través de declaraciones de misión, sanciones, recompensas y políticas impresas. En tanto que los supuestos básicos fundamentales representan aquellas creencias implícitas sobre la forma en que se deben hacer las cosas (Gibson \& Barsade, 2003).

En otras palabras, la cultura organizacional refleja como los miembros de la organización actúan diariamente, enfrentan desafíos y cambios, interactúan entre ellos para alcanzar las metas propuestas, responden al cambio y se presentan frente a clientes y aliados (Howard-Grenville, Lahneman, \& Pek, 2020: 29).

En este marco, se puede también decir que la cultura no se refleja solo en proclamaciones gerenciales, como algunos podrían pensar, pero sobretodo en las prácticas diarias de 
la organización y en el conjunto de herramientas culturales usadas cuando se analizan problemas y se plantean nuevas soluciones. De igual manera, como es sugerido por Howard-Grenville et al, (2020), lo anterior deja entrever a la cultura de una organización como un sistema abierto que se nutre constantemente de sus vínculos internos y con el exterior y aprende de los mismos para impulsar nuevas conductas. Por lo mismo, dependiendo de las prácticas generalmente aceptadas se pueden distinguir diferentes tipos de organizaciones que frente a problemas responden de una $u$ otra manera, por ejemplo, con independencia y emprendimiento o consensos y permisos (Balthazard, Cooke, \& Potter, 2006; Cooke \& Szumal, 2000; Schneider \& Barbera, 2014).

En este contexto, la aplicación del Pensamiento de Diseño puede tener múltiples efectos sobre la cultura organizacional. Por una parte, su uso puede hacer visible los elementos que componen la cultura actualmente, muchos de los cuales generalmente se encuentran en disonancia con lo que asume la gerencia (Howard-Grenville et al, 2020). Por otro lado, su aplicación puede impulsar las capacidades de innovación de la organización a través del uso del razonamiento abductivo, el cual por sus características puede contradecirse a la cultura existente y forzar la creación de nuevas estructuras, procesos, y adaptación de tecnologías, facultando el desarrollo de nuevos sistemas de creencias y valores (Deserti \& Rizzo, 2014).

En relación con esto último, es posible destacar que la aplicación del Pensamiento de Diseño, al tratarse de un enfoque que promueve la interacción, el diálogo y el aprendizaje interdepartamentalmente, con consumidores y con otros actores por fuera de la organización fuerza a los miembros de la misma a desafiar sus límites y dogmas, revisitar sus estrategias, aceptar al fracaso como herramienta de su trabajo, descartar lo esencial de lo intrascendente, asumir riesgos y replantearse su visión estratégica. Esto favorece el desarrollo de enfoques cercanos a las necesidades de su público objetivo, la búsqueda de impacto, y la identificación de oportunidades desde las bases de la firma, y no desde la gerencia general, como usualmente sucede (Deserti \& Rizzo, 2014; Elsbach \& Stigliani, 2018; Liedtka, 2018; Mintzberg, 1989).

En este sentido, se ha observado que el uso constante de herramientas tales como entrevistas y observaciones etnográficas, generalmente usadas durante las etapas de empatía y definición del proceso de Pensamiento de Diseño, contribuyen al desarrollo de culturas organizacionales enfocadas en las necesidades de los usuarios (Liedtka, 2014; Price \& Wrigley, 2016; Seidel \& Fixson, 2013). En tanto que el uso de herramientas y espacios de cogeneración de ideas propician que los equipos se tornen más abiertos a la toma de riesgos y acepten mayor ambigüedad cuando llevan adelante un proyecto (Hoyt \& Sutton, 2017; Wattanasupachoke, 2012). Similarmente, se ha señalado que la construcción de prototipos y evaluación rápida de los mismos puede favorecer el fortalecimiento de dinámicas de experimentación y aceptación de fracasos (Michlewski, 2008; Rauth, Carlgren, \& Elmquist, 2014; Steen, Buijs, \& Williams, 2014).

Empero a lo mencionado, es necesario considerar que lejos de ser un 
proceso inmediato, el pensamiento de diseño se enmarca dentro de un ciclo de aprendizaje continuo, estructurado en la práctica constante del mismo. En otras palabras, a medida que la organización empiece a cambiar, motivada por los procesos y restructuraciones impulsados por el Pensamiento de Diseño, su transformación plena en una organización de cultura de diseño aun requerirá de la reflexión constante de sus resultados y efectividad, y de la promoción a todos los niveles de sus bondades (Elsbach \& Stigliani, 2018). Este esquema será más sencillo en la medida en que la cultura organizacional actual se alinee con los objetivos del Pensamiento de Diseño o se muestre flexible a cambiar como resultado del mismo (Chen \& Venkatesh, 2013; Kolko, 2015; Tischler, 2009; Wilkie, et al, 2010).

En función de lo mencionado, y en comparación con los procedimientos aplicados por cuerpos gerenciales alrededor del mundo en torno al impulso de la innovación a través de reasignación de recursos, modificación de estructuras o cirugías menores en su misión escrita, se puede señalar que el Modelo de Innovación Impulsado por Procesos de Diseño, como el Pensamiento de Diseño, se apalanca del cambio en la filosofía, políticas, y estrategias de los miembros de la organización para favorecer el surgimiento de elementos diferenciadores y ventajas competitivas (Marín, 2004; Naranjo-Valencia, Jiménez-Jiménez, \& Sanz-Valle, 2011).

\section{Cambio Organizacional}

La introducción del pensamiento de diseño en una organización y su utilización como mecanismo de transformación cultural en favor de la innovación requiere de la implementación concertada de acciones y de la planificación bien estructurada de las mismas. Caso contrario, y como lo mencionan Beer (1990) y Velázquez, Cruz, \& Vargas (2018), cualquier intento de desafiar el estatus quo, experimentar con ideas diferentes y explorar nuevas oportunidades puede terminar siendo de corta vida, generar resistencia interna e incluso escepticismo.

Frente a ello, resulta necesario para los gerentes de la organización trabajar en tres dimensiones particulares del cambio organizacional: desarrollo interno, procedimientos de transición y transformación de supuestos (Ackerman, Anderson, \& Marquardt, 1997). El desarrollo interno, como su nombre lo indica, se soporta sobre un enfoque comportamental y sostiene que el cambio en una organización se da a partir del fortalecimiento de capacidades y habilidades de su mano de obra y es apoyado por la restructuración de procesos, de tal manera a que estos contribuyan a la exploración y puesta en marcha de iniciativas no tradicionales por parte de los empleados (Fierro, Benavides, \& Andrade, 2020; Rosenberg et al, 2016). Por otro lado, los procedimientos de transición, buscan encontrar formas que faciliten la adaptación de los miembros de la organización a nuevas prácticas y procedimientos, permitiéndoles identificar las debilidades actuales y las rutas necesarias para alcanzar mejores posiciones estratégicas en el futuro (Schein, 1969). La transformación de supuestos, por su parte, se apoya en el aprendizaje constante y en la definición de nuevos patrones de comportamiento a situaciones habituales (Chan \& Mauborgne, 2005; Gibson \& Barsade, 2003). 
En relación con lo mencionado, una iniciativa que podría fomentar conjuntamente el desarrollo interno y promover una transición adecuada hacia un nuevo orden de trabajo, es la implementación de un equipo de apoyo en diseño. Si bien estudios previos, como el de Dunne (2018), ya han señalado la utilidad de iniciativas como estas por su potencial de crear soluciones diferenciadoras por medio de laboratorios de investigación y desarrollo, es necesario resaltar que la utilidad de estos equipos va más allá, pudiéndose convertir en un eje de innovación para toda la firma. Específicamente, y tal como lo demuestran iniciativas como Equipos-A y Panda Labs del World Wildllife Fund (WWF), los equipos de innovación pueden asumir roles tales como acompañamiento técnico de proyectos en innovación, entrenamiento en el pensamiento de diseño y modelos de negocio, consolidación de indicadores de impacto y monitoreo, priorización estratégica de propuestas, y construcción de comunidades a través de la difusión de avances, logros y lecciones aprendidas con actores clave (World Willife Fund, 2020).

De igual manera, otro elemento que se ajusta al trabajo en pro del desarrollo interno y transición corresponde a la creación de una ruta de ideas ${ }^{2}$, también entendida como un proceso estructurado para identificar un problema, validarlo, verificar su prioridad estratégica, construir un planteamiento de solución, y lanzarlo al mercado (Sahni, et al, 2017). Si bien la misma podría parecer un proceso similar al encontrado en el modelo de fases y puertas en lo referente a la existencia de etapas (Cooper, 2008), es necesario diferenciar que el mismo involucra una reconversión en el modo de gestionar proyectos en las organizaciones, a través de la simplificación de procesos, desconcentración de poderes administrativos, incubación de ideas, y co creación de soluciones con actores externos (i.e., innovación abierta). En contraposición con proyectos ordinarios, la ruta de ideas permite dar a los proyectos de innovación el suficiente espacio para que los equipos a cargo de ellos se equivoquen, aprendan, obtengan retroalimentación rápida $y$ puedan fortalecer a la organización como un todo, trayendo conocimiento y recursos de aliados (Chesbrough, 2003; Miller, 2011).

En cuanto a la transformación de supuestos, se distingue especialmente al liderazgo catalítico y a sus derivaciones como los mecanismos más efectivos de implementación (Snyder, Ingelsson, \& Bäckström, 2018). Esto se debe a que el proceder de los líderes envía señales al resto de la organización acerca de lo que es impulsado y aceptado frente a cursos de acción determinados (Cooke \& Szumal, 2000; Liker, 2004; Sathe, 1985). Si los líderes, por ejemplo, promueven activamente el diagnósticos cuidadoso de problemas y crean un atmosfera organizacional donde los fracasos son aceptados y no castigados, poco a poco este modo de trabajo comenzará a permear en los otros empleados (Sanchez, 2006). En el mismo sentido, y yendo un paso más allá, se puede

2 Innovation pathways en Ingles. 
decir que en la medida en que los líderes dispongan recursos financieros, técnicos y de tiempo, y fomenten el libre intercambio de ideas entre empleados, sin importar su cargo, democratizarán la cultura de diseño, fomentando el uso constante de las herramientas existentes y promoviendo el desarrollo de nuevas.

\section{Limitaciones al cambio organizacional}

El cambio organizacional promovido por el Modelo de Innovación de Procesos en Diseño puede enfrentar en su implementación algunas limitaciones sobre cuya existencia es necesario tomar conocimiento. De acuerdo con Rosenberg et al, (2016), estas son: 1) zona de confort corporativa, 2) defensas internas y 3) miopía institucional. La zona confort se refiere a la situación en la que por miedos a lo nuevo se termina rechazando algo que podría ser significativamente mejor. Esta zona de confort se fortalece especialmente en circunstancias en que los miembros de una organización no reciben suficiente información o instrucciones previas pero se les demanda la ejecución de nuevos procesos, frente a lo cual naturalmente responden con escepticismo y se amparan en los métodos conocidos (Ford, Ford, \& D'Amelio, 2008; Suciu \& Baughn, 2016). Las defensas internas, por otro lado, describen la existencia de mecanismos de control y seguimiento bajo los cuales se mide el rendimiento y grado de ejecución de los proyectos. En este contexto, al tomar mayor tiempo de implementación, las metodologías de trabajo alternativas pueden ser indirectamente rechazadas (Hoag, Ritschard, \& Cooper, 2002). Finalmente, la miopía institucional parte de la falta de visión y miedo al fracaso, las cuales pueden impedir explorar nuevos modelos de negocio bajo argumentos tales como altos costos corporativos, menores probabilidades de éxito o restricciones por elementos externos (Edwards, 2012).

\section{Conclusiones}

En un mundo caracterizado por constantes desafíos en donde el surgimiento de nuevos competidores y el cambio rápido de necesidades de los consumidores imponen a las organizaciones reconstruirse a sí mismas, el Modelo de Innovación impulsado por Procesos de Diseño cobra mayor relevancia que nunca. La razón para ello parte de que contrariamente a las nociones gerenciales que se tienen en cuanto a la innovación como algo adquirible y anexable a una organización por medio de tecnología, esta surge y puede mantenerse en el tiempo solo como resultado de un cambio de filosofía en favor de mayor exploración de problemas, identificación de necesidades, intercambio de conocimiento y toma de riesgos.

En este contexto, y como se demostró a lo largo de este documento, resulta pertinente que las organizaciones impulsen internamente proyectos orientados a la utilización constante del Pensamiento de Diseño. El mismo, a través de sus metodologías y procesos, puede ayudarles no solo a canalizar mejor los problemas que identifiquen y las soluciones que generen, pero sentar las bases para que la organización como un todo cambie, a través del impulso a procesos más flexibles, desconcentración de poderes, y restructuración de modos de trabajo. Es necesario, sin embargo, también 
reconocer que la implementación de un nuevo modelo de innovación necesita de la edificación de estructuras internas de apoyo, ya sea a través de la formulación de equipos que provean acompañamiento técnico, de la creación de guías de procesos o el establecimiento de nuevos modelos de liderazgo, ya que estos elementos pueden mitigar limitaciones, dar legitimidad a iniciativas no tradicionales, y promover nuevos patrones comportamentales en favor de la innovación disruptiva.

\section{Referencias Bibliográficas}

Ackerman, L., Anderson, D., \& Marquardt, M. (1997). Development, transition or transformation: Bringing change leadership into the 21st century. $O D$ Practitioner, 28(4), 5-16.

Balthazard, P. A., Cooke, R. A., \& Potter, R. E. (2006). Dysfunctional culture, dysfunctional organization: Capturing the behavioral norms that form organizational culture and drive performance. Journal of Managerial Psychology, 21(8), 709-732. https://doi. org/10.1108/02683940610713253

Beer, M. (1990). Por Qué los Programas de Cambio no se Traducen en Cambios. Harvard Business Review, 68(6).

Boland Jr, R. J., Collopy, F., Lyytinen, K., \& Yoo, Y. (2008). Managing as designing: lessons for organization leaders from the design practice of Frank O. Gehry. Design Issues, 24(1), 10-25.

Brown, T., \& Wyatt, J. (2010a). Design thinking for social innovation. Development Outreach, 12(1), 2943.

Brown, T., \& Wyatt, J. (2010b). Design Thinking for Social Innovation.
Development Outreach, 12(1), 2943. Recuperado de www.ssireview. com

Buchanan, R. (2015). Worlds in the Making: Design, Management, and the Reform of Organizational Culture. She Ji, 1(1), 5-21. https:// doi.org/10.1016/j.sheji.2015.09.003

Chan, W., \& Mauborgne, R. (2005). La estrategia del océano azul: cómo crear en el mercado espacios no disputados en los que la competencia sea irrelevante. Barcelona: Ediciones Granica.

Chang, Y., Kim, J., \& Joo, J. (2013). An exploratory study on the evolution of design thinking: Comparison of Apple and Samsung. Design Management Journal, 8(1), 22-34.

Chen, S., \& Venkatesh, A. (2013). An investigation of how design-oriented organisations implement design thinking. Journal of Marketing Management, 29(15-16), 16801700. https://doi.org/10.1080/026725 7x.2013.800898

Chesbrough, H. W. (2003). Open innovation: The new imperative for creating and profiting from technology. Harvard Business Press.

Chiva, R., \& Alegre, J. (2009). Investment in Design and Firm Performance: The Mediating Role of Design Management. The Journal of Product Innovation Management, 26(4), 424440. https://doi.org/10.1111/j.15405885.2009.00669.x

Cooke, R. A., \& Szumal, J. L. (2000). Using the organizational culture inventory to understand the operating cultures of organizations. Handbook of Organizational Culture and Climate, 4, 1032-1045.

Cooper, R. G. (2008). Perspective: The stage-gates ${ }^{\circledR}$ idea-to-launch 
process - Update, what's new, and NexGen systems. Journal of Product Innovation Management, 25(3), 213232. https://doi.org/10.1111/j.15405885.2008.00296.x

Deserti, A., \& Rizzo, F. (2014). Design and the Cultures of Enterprises. Design Issues, 30(1), 36-56.

Dorst, K., \& Cross, N. (2001). Creativity in the design process: co-evolution of problem-solution. Design Studies, 22(5), 425-437. https://doi. org/10.1016/s0142-694x(01)00009-6

Dunne, D. (2011). User-centred Design and Design-centred Business School. In R. Buchanan, S. Junginger, \& T. Lockwood (Eds.), The handbook of design management (pp. 128-143). Berg, London, UK: Bloomsbury Academic.

Dunne, D. (2018). Implementing design thinking in organizations: an exploratory study. Journal of Organization Design, 7(1). https:// doi.org/10.1186/s41469-018-0040-7

Edwards, D. (2012). Innovation adventures beyond the core. Research-Technology Management, 55(6), 33-41.

Elsbach, K. D., \& Stigliani, I. (2018). Design Thinking and Organizational Culture: A Review and Framework for Future Research. Journal of Management, 44(6), 2274-2306. https://doi. org/10.1177/0149206317744252

Escorsa, P., \& Pasola, J. (2004). Tecnología e innovación en la empresa (Vol. 148). Univ. Politèc. de Catalunya.

Fierro, F. A., Benavides, D., \& Andrade, J. M. (2020). Cambio organizacional: una visión teórica de su evolución conceptual. Revista Venezolana de Gerencia (RVG), 25(4), 131-147.

Filippetti, A. (2011). Innovation modes and design as a source of innovation: a firm-level analysis. European Journal of Innovation Management, 14(1), 5-26. https:// doi.org/10.1108/14601061111104670

Ford, J. D., Ford, L. W., \& D'Amelio, A. (2008). Resistance to Change: The Rest of the Story. The Academy of Management Review, 33(2), 362-377. https://doi.org/10.5465/ amr.2008.31193235

Gibson, D. E., \& Barsade, S. G. (2003). Managing organizational culture change: The case of long-term care. Journal of Social Work in Long Term Care, 2(1-2), 11-34. https://doi. org/10.1300/J181v02n01 02

Glen, R., Suciu, C., Baughn, C. C., \& Anson, R. (2015). Teaching design thinking in business schools. The International Journal of Management Education, 13(2), 182-192.

Hernández, R., Fernández, C., Baptista, P., Méndez, S., \& Mendoza, C. (1998). Metodología de la investigación (Vol. 1). Mcgraw-hill México, DF.

Hoag, B., Ritschard, H., \& Cooper, C. (2002). Obstacles to effective organizational change: the underlying reasons. Leadership \& Organization Development, 23(1), 6-15.

Howard-Grenville, J., Lahneman, B., \& Pek, S. (2020). Organizational Culture as a TOOL for CHANGE. Stanford Social Innovation Review, 18(3), 28-33.

Hoyt, D., \& Sutton, R. I. (2017). What design thinking is doing for the San Francisco Opera. https://hbr. org/2016/06/what-design-thinking-isdoing-for-the-san-francisco-opera

Jayakumar, T., Das, K., \& Srivastava, N. (2019). Design thinking: a working strategy for the third sector. Journal of Business Strategy, 40(5), 28-38. 
Montenegro Orozco, Josué Carlos

Innovación y Diseño: Como transformar organizaciones y prosperar en mercados competitivos

Junginger, S. (2008). Product Development as a Vehicle for Organizational Change. Design Issues, 24(1), 26-35. https://doi. org/10.1162/desi.2008.24.1.26

Koen, P. (2015). Lean startup in large enterprises using human-centered design thinking: a new approach for developing transformational and disruptive innovations. https://doi.org/ Koen, Peter, Lean Startup in Large Enterprises Using Human-Centered Design Thinking: A New Approach for Developing Transformational and Disruptive Innovations (January 27, 2015). http://dx.doi.org/10.2139/ ssrn.2556187

Kolko, J. (2015). Design thinking comes of age. Harvard Business Review, (September). Recuperado de https:// hbr.org/2015/09/design-thinkingcomes-of-age

Kong, E. (2010). Innovation processes in social enterprises: An IC perspective. Journal of Intellectual Capital, 11(2), 158-178. https://doi. org/10.1108/14691931011039660

Kong, E., \& Thomson, S. B. (2009). An intellectual capital perspective of human resource strategies and practices. Knowledge Management Research \& Practice, 7(4), 356-364.

Lawson, B. (2006). How designers think: The design process demystified. Routledge.

Liedtka, J. (2014). Innovative ways companies are using design thinking. Strategy \& Leadership, 42(2), 40-45. https://doi.org/10.1108/SL-01-20140004

Liedtka, J. (2018). Why design thinking works. Harvard Business Review, 96(5), 72-79.

Liedtka, J., \& Ogilvie, T. (2011). Designing for growth: A design thinking tool kit for managers. Columbia University Press.

Liker, J. K. (2004). Toyota way: 14 management principles from the world's greatest manufacturer. McGraw-Hill.

Make Sense \& Schoolab (2020, 22 de Mayo). Webinar a Cause Design Thinking \& Lean Startup 101. https:/levents.makesense. org/en/e/webinar-designthinking-and-lean-startup-1015eb4e8aedf5c97058d6f17fc

Marín, H. (2004). La gerencia del cambio en contextos de globalización The Management of Change in the Context of Globalization. Revista de Ciencias Sociales (RCS), X(1), 9-27.

Marr, B., \& Roos, G. (2005). A strategy perspective on intellectual capital. In Perspectives on intelectual capital: Multi-disciplinary insights into management, measurement, and reporting (pp. 28-52). Amsterdam: Elsevier Butterworth-Heinemann.

Martin, R. L. (2009). The design of business : why design thinking is the next competitive advantage. Harvard Business Press.

Michlewski, K. (2008). Uncovering Design Attitude: Inside the Culture of Designers. Organization Studies, 29(3), 373-392. https://doi. org/10.1177/0170840607088019

Miller, D. (2011). Miller (1983) revisited: $A$ reflection on EO research and some suggestions for the future. Entrepreneurship Theory and Practice, 35(5), 873-894.

Mintzberg, H. (1989). Mintzberg on management: Inside our strange world of organizations. Simon and Schuster.

Naranjo-Valencia, J. C., JiménezJiménez, D., \& Sanz-Valle, R. (2011). 
Innovation or imitation? The role of organizational culture. Management Decision, 49(1), 55-72. https://doi. org/10.1108/00251741111094437

Organisation for Economic Cooperation and Development. (2015). The innovation imperative in the public sector: Setting an agenda for action. OECD Publishing.

Perks, H., Cooper, R., \& Jones, C. (2005). Characterizing the Role of Design in New Product Development: An Empirically Derived Taxonomy. The Journal of Product Innovation Management, 22(2), 111-127. https://doi.org/10.1111/j.0737$\underline{6782.2005 .00109 . x}$

Porter, M. (2007). La ventaja competitiva de las naciones. Harvard Business Review, 85(11), 69-95.

Price, R., \& Wrigley, C. (2016). Design and a Deep Customer Insight Approach to Innovation. Journal of International Consumer Marketing, 28(2), 92-105. https://doi.org/10.108 $\underline{0 / 08961530.2015 .1092405}$

Rauth, I., Carlgren, L., \& Elmquist, M. (2014). Making It Happen: Legitimizing Design Thinking in Large Organizations. Design Management Journal, 9(1), 47-60. https://doi. org/10.1111/dmj.12015

Rizzo, F., Deserti, A., \& Cobanli, O. (2017). Introducing Design Thinking in Social Innovation and in the Public Sector: A design based learning framework. European Public \& Social Innovation Review (EPSIR), 2(1).

Rosenberg, N., Chauvet, M.-C., \& Kleinman, J. (2016). Leading for a Corporate Culture of Design Thinking: Achieve cultural transformation-and breakthrough results-by embedding design thinking into your company's DNA. Insigniam Quarterly, 61-75. Recuperado de https://quarterly. insigniam.com/corporate-culture/ leading-for-a-corporate-culture-ofdesign-thinking/

Sahni, N., Lanzerotti, L., Bliss, A., \& Pike, D. (2017). Is Your Nonprofit Built for Sustained Innovation? Six useful starting points for nonprofits that want to build their capacity to continuously innovate. https://ssir. org/articles/entry/is your nonprofit built for sustained innovation\#

Sanchez, R. (2006). Integrating Design into Strategic Management Processes. Design Management Review, 17(4), 10-17. https://doi. org/10.1111/j.1948-7169.2006. $\underline{\text { tb00057.x }}$

Sanders, E. B. N. (2002). From usercentered to participatory design approaches. In J. Frascara (Ed.), Design and the social sciences: Making connections. Taylor \& Francis Books Limited.

Sathe, V. (1985). Culture and related corporate realities: Text, cases, and readings on organizational entry, establishment, and change. Richard D Irwin.

Schein, E. H. (1969). Process consultation: its role in organization development. Reading, Mass. : Addison-Wesley Pub. Co.

Schein, E. H. (2017). Organizational culture and leadership (5th Edition). Hoboken, New Jersey : Wiley.

Schein, E. H., \& Schein, P. A. (2019). The corporate culture survival guide. San Francisco: John Wiley \& Sons.

Schneider, B., \& Barbera, K. M. (2014). Introduction: The Oxford handbook of organizational climate and culture. In B. Schneider \& K. M. Barbera (Eds.), The Oxford handbook of organizational climate and culture. (pp. 3-20). https://doi.org/10.1093/ 
Montenegro Orozco, Josué Carlos

Innovación y Diseño: Como transformar organizaciones y prosperar en mercados competitivos

\section{oxfordhb/9780199860715.013.0001}

Schumpeter, J. A., \& Arrarte, J. P. (1976). Teoría del desenvolvimiento económico: una investigación sobre ganancias, capital, crédito, interés y ciclo económico. Fondo de cultura económica México.

Seidel, V. P., \& Fixson, S. K. (2013). Adopting Design Thinking in Novice Multidisciplinary Teams: The Application and Limits of Design Methods and Reflexive Practices: Adopting Design Thinking in Novice Teams. The Journal of Product Innovation Management, 30, 19-33. https://doi.org/10.1111/jpim.12061

Snyder, K., Ingelsson, P., \& Bäckström, I. (2018). Using design thinking to support value-based leadership for sustainable quality development. Business Process Management Journal, 24(6), 1289-1301. https:// doi.org/10.1108/BPMJ-02-2018-0045

Steen, M., Buijs, J., \& Williams, D. (2014). The Role of Scenarios and Demonstrators in Promoting Shared Understanding in Innovation Projects. International Journal of Innovation and Technology Management, 11(1), 1440001. https://doi.org/10.1142/ S021987701440001X

Suciu, C., \& Baughn, C. (2016). Design Thinking and Organizational Change: Developing a Human-Centered Culture. European Conference on Innovation and Entrepreneurship, 787. Academic Conferences International Limited.

Sutton, R. I., \& Hargadon, A. (1996). Brainstorming Groups in Context: Effectiveness in a Product Design
Firm. Administrative Science Quarterly, 41(4), 685-718. https:// doi.org/10.2307/2393872

Tischler, L. (2009). Ideo's David Kelley on design thinking. https://www. fastcompany.com/1139331/ideosdavid-kelley-design-thinking

Vega, V., Ferro, H., Ruiz, M., \& Bonomie, M. (2020). Innovación y éxito empresarial: algunas reflexiones teóricas. Revista Venezolana de Gerencia (RVG), 25(91), 938-953.

Velázquez, J., Cruz, E., \& Vargas, E. (2018). Cooperación empresarial para el fomento de la innovación en la pyme turística. Revista de Ciencias Sociales (RCS), XXIV(3), 9-20.

Wattanasupachoke, T. (2012). Design Thinking, Innovativeness and Performance: An Empirical Examination. International Journal of Management and Innovation, 4(1), 1.

Wilkie, A., Gaver, W., Hemment, D., \& Giannachi, G. (2010). Creative Assemblages: Organisation and Outputs of Practice-Led Research. Leonardo (Oxford), 43(1), 98$99 . \quad$ https://doi.org/10.1162/ leon.2010.43.1.98

World Willife Fund (2020). What is Panda Labs?. https://wwf.panda.org/ projects/panda labs/?

Wyrwicka, M. K., \& Chuda, A. (2019). The diagnosis of organizational culture as a change's factor in the context application of design thinking. Logforum, 15(2), 279-290. https:// doi.org/10.17270/J.LOG.2019.319 